\title{
¿Cómo desarrollamos la memoria de un proyecto constructivo de infraestructura aeroportuaria?
}

\section{How do we develop the memory of an airport infrastructure construction project?}

JORGE FERNÁNDEZ DE LA CRUZ

ORCID: https://orcid.org/0000-0002-3677-1849

Universidad de Sevilla

Departamento de Ingeniería de la

Construcción y Proyectos de Ingeniería

jfernandez2@us.es

Fecha de recepción: 12-11-2019

Fecha de aceptación: 26-11-2019

DOI: http://dx.doi.org/10.12795/9788447221912.083

Pp.: 1889-1906 


\section{Resumen}

El ciclo de mejora realizado en el 2018/2019 ha complementado al Ciclo de Mejora en el Aula (CIMA) realizado en el curso 2017/2018. La innovación docente se ha dirigido hacia la potenciación de aspectos que están poco presentes en los alumnos y que están dirigidos principalmente a desarrollar su capacidad de análisis, de asunción de responsabilidades, de trabajar en equipo, de gestionar recursos, de tomar de decisiones, y de la interacción personal con equipos y grupos de trabajo.

El motivo de la mejora y continuación del CIMA anterior es la observación de la dificultad que tienen los estudiantes en su implicación y en su participación en el desarrollo de la clase.

La innovación desarrollada da respuesta a las necesidades y dificultades analizadas, profundizando en la participación e implicación de los estudiantes en su proceso de enseñanza-aprendizaje tomando como punto de referencia el proyecto de infraestructuras aeroportuarias.

Palabras clave: Ingeniería de la construcción y proyectos de ingeniería, Grado de Ingeniería Aeroespacial, docencia universitaria, experimentación docente universitaria, infraestructuras aeroportuarias, Implicación y participación activa.

\section{Abstract}

The improvement cycle carried out in 2018/2019 has complemented the Classroom Improvement Cycle carried out in the 2017/2018 academic year. Teaching innovation has been directed towards the empowerment of aspects that are not very present in students and that are mainly aimed at developing their capacity for analysis, assuming responsibilities, working in teams, managing resources, making decisions, and of personal interaction with teams and work groups.

The reason for the improvement and continuation of the previous ICIC is the observation of the difficulty that students have in their involvement and in their active participation in the development of the class. The innovation developed responds to the needs and difficulties analyzed, deepening the participation and involvement of students in their teaching-learning process, taking as a point of reference the airport infrastructure project.

Keywords: "Construction engineering and engineering projects", "Aerospace Engineering Degree", "university teaching", "university teaching experimentation", "airport infrastructure", "Involvement and active participation". 


\section{Contexto del ciclo de mejora}

La asignatura de Proyectos del Grado de Ingeniería Aeroespacial es casi el primer contacto que tienen muchos alumnos del Grado con las infraestructuras aeroportuarias, estando un poco denostado el tema aeroportuario si lo comparamos con otras materias del grado. La asignatura pretende que los estudiantes conozcan el ámbito aeroportuario que también relaciona aspectos de la aeronáutica. Para el desarrollo del CIMA se dispone de un aula equipada y los estudiantes disponen de una bibliografia (accesible a través de páginas web) para consulta y que les sirve de base para las tareas que se van a realizar. A su vez se establece la creación de un disco virtual para gestionar la bibliografía indicada y otras de interés, a la vez que se gestiona la documentación generada en el desarrollo del CIMA.

Los objetivos del CIMA para el curso 2018/2019 dirigidos a los estudiantes se resumen en los siguientes puntos:

- Adquirir conocimientos específicos en el ámbito del entorno aeroportuario, así como mejorar el interés por esta rama de la Ingeniera Aeronáutica.

- Fomentar el trabajo en equipo a través de la organización y gestión de los recursos existentes.

- Fomentar el espíritu crítico y la capacidad de análisis ante las situaciones planteadas con la finalidad de plantear propuestas para valorar y tomar las decisiones que se consideren adecuadas en función del análisis realizado.

- Fomentar la toma de decisiones en función del análisis crítico que se ha establecido.

- Estar habituado a situaciones de exposiciones en público.

En el proceso de innovación y mejora docente mantengo, en general, los principios didácticos del curso 2017/18: 
- Desde el punto de vista de los contenidos se pretende englobar dos tipos de contenidos: el relacionado con el alcance del trabajo y el que se centra en los contenidos transversales, según el CIMA del curso 2017/18 (Fernández de la Cruz, 2017).

- En relación con la metodología: la importancia de partir de preguntas y/o problemas que generen interés en los estudiantes, el fomento del debate para compartir conocimientos, aplicaciones y dudas en un clima de trabajo colaborativo, el desarrollo del aprendizaje de los estudiantes mediante materiales docentes que sirvan de guía para la resolución de los problemas planteados según el CIMA del curso 2017/18 (Fernández de la Cruz, 2017).

- En la evaluación de los estudiantes: la participación e implicación en clase de los estudiantes en la resolución de los problemas planteados según el CIMA del curso 2017/18 (Fernández de la Cruz, 2017).

Con objeto de alcanzar los objetivos expresados para el curso 2018/2019, incorporo el siguiente principio didáctico:

- En el proceso de enseñanza-aprendizaje es necesario que los estudiantes se impliquen de una manera más intensa en el desarrollo del trabajo y que ese interés repercuta en el beneficio general de la clase y de los propios alumnos.

\section{Diseño previo}

\section{Mapas de contenidos y preguntas clave}

En siguiente figura queda resumido el mapa de contenidos y preguntas clave. 


\begin{tabular}{|c|c|c|}
\hline $\begin{array}{c}\text { ELEMENTOS } \\
\text { CONSTRUCTIVOS }\end{array}$ & \multirow{4}{*}{$\begin{array}{l}\text { INFRAESTRUCTURA } \\
\text { AEROPORTUARIA }\end{array}$} & ÁMBITO ACACÉMICO \\
\hline ¿Cómo participo? & & $\begin{array}{l}\text { ¿Qué conozco? } \\
\text { ¿Qué aprendizaje necesito? }\end{array}$ \\
\hline RECURSOS HUMANOS & & RECURSOS MATERIALES \\
\hline ¿Cómo los gestiono? & & $\begin{array}{l}\text { ¿Cómo los gestiono? } \\
\text { ¿Qué necesito? }\end{array}$ \\
\hline ¿Qué aporto desde mi persona? & \multirow{9}{*}{$\begin{array}{c}\text { MEMORIA DE } \\
\text { PROYECTO } \\
\text { ¿Cómo estructuro el } \\
\text { proyecto? }\end{array}$} & ¿Cómo organizo el proyecto? \\
\hline \multirow[t]{2}{*}{ ¿Qué aporto desde la técnica? } & & $\begin{array}{l}\text { ¿Qué tareas son necesarias? } \\
\text { ¿Quién las realiza? }\end{array}$ \\
\hline & & \\
\hline $\begin{array}{l}\text { ¿Qué aporto desde mi competencia } \\
\text { profesional? }\end{array}$ & & ¿Qué controles realizo? \\
\hline \multirow[t]{2}{*}{$\begin{array}{l}\text { ¿Cómo suplo mis carencias en mi } \\
\text { campo? }\end{array}$} & & ¿Qué dificultades encuentro? \\
\hline & & ¿Qué puedo realizar? \\
\hline \multirow[t]{2}{*}{$\begin{array}{l}\text { ¿Cómo interactúo dentro de mi } \\
\text { área de trabajo? }\end{array}$} & & ¿Cómo las puedo resolver? \\
\hline & & ¿Con quién debo contactar? \\
\hline $\begin{array}{l}\text { ¿Cómo interactúo en otras áreas de } \\
\text { trabajo? }\end{array}$ & & $\begin{array}{l}\text { ¿Qué está en } \mathrm{mi} \\
\text { campo/competencia? }\end{array}$ \\
\hline
\end{tabular}

Figura 1. Mapa de contenidos y preguntas clave

\section{Modelo metodológico previo}

En el desarrollo del curso 2017/2018 se siguió el esquema expresado en la tabla siguiente.

Tabla 1. Estructura de sesión estándar

\begin{tabular}{|c|l|c|}
\hline \multicolumn{3}{|c|}{ Modelo de Sesión estándar } \\
\hline Concepto & \multicolumn{1}{|c|}{ Descripción } & $\begin{array}{c}\text { Tiempo } \\
\text { estimado }\end{array}$ \\
\hline $\begin{array}{c}\text { Introducción } \\
(\mathrm{I})\end{array}$ & $\begin{array}{l}\text { Saludo. Exposición del problema } \\
\text { o revisión del acta generada en la } \\
\text { sesión anterior }\end{array}$ & 10 minutos \\
\hline
\end{tabular}

Jornadas de Formación e Innovación Docente del Profesorado I № 2 (2019) Esta obra se distribuye con la licencia Creative Commons Reconocimiento-NoComercial-SinObraDerivada Internacional (CC BY-NC-ND 4.0.) 


\begin{tabular}{|c|c|c|}
\hline $\begin{array}{l}\text { Hipótesis Inicial } \\
\qquad(\mathrm{H} \mathrm{I})\end{array}$ & $\begin{array}{l}\text { Se plantea la hipótesis inicial } \\
\text { del trabajo sobre la que se va a } \\
\text { trabajar en clase }\end{array}$ & 5 minutos \\
\hline $\begin{array}{l}\text { Hipótesis } \\
(\mathrm{H} 1, \mathrm{H} 2, \mathrm{H} 3)\end{array}$ & $\begin{array}{l}\text { Cada grupo de clase desarrolla su } \\
\text { hipótesis de los contenidos que le } \\
\text { corresponden. Trabajan en el aula } \\
\text { y el profesor les apoya y guía en } \\
\text { el establecimiento de sus propias } \\
\text { hipótesis }\end{array}$ & 25 minutos \\
\hline $\begin{array}{l}\text { Puesta en Común } \\
\qquad(P C 1)\end{array}$ & $\begin{array}{l}\text { Se realiza la puesta en común } \\
(P C) \text { de todas las hipótesis, para } \\
\text { analizarlas y debatirlas }\end{array}$ & 20 minutos \\
\hline $\begin{array}{l}\text { Hipótesis } \\
(\mathrm{H} 4, \mathrm{H} 5, \mathrm{H} 6)\end{array}$ & $\begin{array}{l}\text { Con todo lo debatido y acordado } \\
\text { en la puesta en común (PC) se } \\
\text { vuelve a trabajar en las hipótesis. } \\
\text { Se pretende que los estudiantes } \\
\text { vayan avanzando y cerrando } \\
\text { asuntos del trabajo propuesto }\end{array}$ & 25 minutos \\
\hline $\begin{array}{l}\text { Puesta en Común } \\
\text { (C 2) }\end{array}$ & $\begin{array}{l}\text { Se realiza la puesta en común } \\
(P C) \text { de todas las hipótesis, para } \\
\text { analizarlas y debatirlas }\end{array}$ & 20 minutos \\
\hline $\begin{array}{c}\text { Tareas Casa } \\
\text { (TC) }\end{array}$ & $\begin{array}{l}\text { Se obtienen las conclusiones de } \\
\text { la puesta en común (PC2) y se } \\
\text { plantean las tareas para realizar } \\
\text { en casa (TC) para la próxima sesión } \\
\text { de clase }\end{array}$ & 15 minutos \\
\hline
\end{tabular}

El desarrollo del CIMA del curso 2017/2018 me llevó a las siguientes consideraciones:

- Se observó que el material que entregó el profesor fue de gran relevancia para un mejor entendimiento y comprensión por los alumnos para la problemática planteada, pero se constató que los alumnos no profundizaron en el alcance del material que repercute en la resolución del proyecto de infraestructuras aeroportuario.

- Se observó que era necesario ahondar en los aspectos de análisis de la documentación facilitada y de referencia. Se debe mejorar el análisis para que sirva de base en el desarrollo del proyecto de infraestructuras aeroportuario. 


\section{Modelo metodológico habitual}

El modelo metodológico habitual se resume en la siguiente tabla.

Tabla 2. Estructura de actividades del modelo metodológico habitual

\begin{tabular}{|l|l|}
\hline \multicolumn{2}{|c|}{ Estructura del ciclo de mejora } \\
\hline Actividades de iniciación (I) & $\begin{array}{l}\text { Exposición del problema o re- } \\
\text { visión del acta de hipótesis ge- } \\
\text { neradas en la sesión anterior }\end{array}$ \\
\hline Actividades de iniciación (II) & $\begin{array}{l}\text { Exposición de hipótesis inicial } \\
\text { de trabajo }\end{array}$ \\
\hline $\begin{array}{l}\text { Actividades de profundiza- } \\
\text { ción (I) }\end{array}$ & $\begin{array}{l}\text { Análisis del problema y es- } \\
\text { tablecimiento de nuevas } \\
\text { hipótesis }\end{array}$ \\
\hline $\begin{array}{l}\text { Actividades de identifica- } \\
\text { ción de dificultades (I) }\end{array}$ & $\begin{array}{l}\text { Puesta en común de las hipó- } \\
\text { tesis identificadas. Análisis y } \\
\text { debate. }\end{array}$ \\
\hline $\begin{array}{l}\text { Actividades de profundiza- } \\
\text { ción (II) }\end{array}$ & $\begin{array}{l}\text { Nuevo análisis y estableci- } \\
\text { miento de nuevas hipótesis }\end{array}$ \\
\hline $\begin{array}{l}\text { Actividades de identifica- } \\
\text { ción de dificultades (II) }\end{array}$ & $\begin{array}{l}\text { Puesta en común de las hipó- } \\
\text { tesis identificadas. Análisis y } \\
\text { debate. }\end{array}$ \\
\hline $\begin{array}{l}\text { Actividades de cierre. } \\
\text { Programación de tareas } \\
\text { para casa }\end{array}$ & $\begin{array}{l}\text { Obtención de conclusiones. Le- } \\
\text { vantado de Acta. } \\
\text { Programación de tareas para } \\
\text { trabajo en casa. } \\
\text { Programación de tareas para la } \\
\text { siguiente sesión. }\end{array}$ \\
\hline
\end{tabular}

Jornadas de Formación e Innovación Docente del Profesorado | № 2 (2019) Esta obra se distribuye con la licencia Creative Commons Reconocimiento-NoComercial-SinObraDerivada Internacional (CC BY-NC-ND 4.0.) 


\section{Modelo metodológico ideal}

El modelo metodológico ideal se dirige hacia la consecución de los objetivos marcados para el desarrollo de la secuencia de los ciclos de innovación docente.

El modelo ideal continúa con las premisas establecidas para la parte práctica de la asignatura, desarrolladas en el curso 2017/2018, e incorpora nuevos valores a alcanzar por los estudiantes en la asignatura:

- Que los estudiantes generen sus propias hipótesis respecto al problema de forma autónoma.

- Que los estudiantes participen de forma activa y aumenten el grado de implicación en la asignatura y en los problemas planteados en el aula.

- Que los estudiantes desarrollen dinámicas de equipo e interactúen entre sí y con las tareas propuestas.

\section{Modelo metodológico posible}

El modelo metodológico posible tiene en consideración las limitaciones del conocimiento de los alumnos en el ámbito del proyecto, así como el grado de interés e implicación que muestran los estudiantes por la asignatura al considerarla fácilmente superable con la participación en clase. Éstos son los principales hándicaps que se encuentran en la asignatura desde mi punto de vista y para solucionarlo estimo que se requiere una dedicación más específica del profesor para potenciar aspectos que permitan que los alumnos muestren mayor interés por la asignatura y se "olviden" en la medida de lo posible de la sola necesidad de aprobar y que se impliquen más en el desarrollo de la asignatura.

Se plantea la siguiente "distribución tipo de actividades" dentro del modelo metodológico posible: 
Tabla 3. Estructura de sesión estándar "posible"

\begin{tabular}{|c|c|c|c|}
\hline Concepto & Descripción & $\begin{array}{l}\text { Tiempo } \\
\text { estimado }\end{array}$ & $\begin{array}{c}\text { Tiempo } \\
\text { acumulado }\end{array}$ \\
\hline$\frac{1}{\text { (Introducción) }}$ & 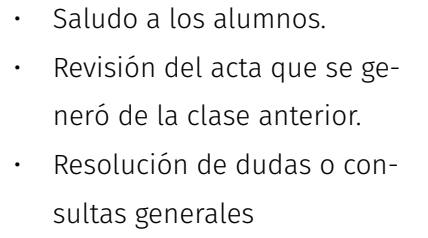 & 5 minutos & 5 minutos \\
\hline $\begin{array}{c}\text { E I } \\
\text { (Explicación } \\
\text { Inicial del ciclo) }\end{array}$ & $\begin{array}{l}\text { Exposición de la temática, di- } \\
\text { námica y calendario del ciclo } \\
\text { de mejora. } \\
\text { - Exposición y planteamiento } \\
\text { de hipótesis inicial del tra- } \\
\text { bajo sobre la que se va a tra- } \\
\text { bajar en clase. } \\
\text { Exposición de documento } \\
\text { base sobre la que se va a } \\
\text { trabajar. El documento de } \\
\text { necesidades (hoja de tra- } \\
\text { bajo) ha de servir de base } \\
\text { para resolver la problemática } \\
\text { planteada }\end{array}$ & 20 minutos & 25 minutos \\
\hline $\begin{array}{c}\mathrm{Cl} \\
\text { (Presuntas del } \\
\text { cuestionario) }\end{array}$ & $\begin{array}{l}\text { Exposición de las pregun- } \\
\text { tas del cuestionario. Identi- } \\
\text { ficación de las preguntas de } \\
\text { trabajo y relación con los as- } \\
\text { pectos relevantes del mapa } \\
\text { de contenidos }\end{array}$ & 15 minutos & 40 minutos \\
\hline $\begin{array}{c}\text { TG } 1 \\
\text { (Trabajo en } \\
\text { Grupo) }\end{array}$ & $\begin{array}{l}\text { - } \text { Trabajo en grupo. } \\
\text { trabajo asignado. } \\
\text { - Trabajo en el aula. Apoyo } \\
\text { y guía del profesor y de la } \\
\text { clase en el desarrollo del tra- } \\
\text { bajo propuesto sobre la base } \\
\text { de los recursos facilitados. }\end{array}$ & 30 minutos & 70 minutos \\
\hline
\end{tabular}




\begin{tabular}{|c|c|c|c|}
\hline $\begin{array}{c}\text { I C } 1 \\
\text { (Intervenciones } \\
\text { de Contraste) }\end{array}$ & $\begin{array}{l}\text { El profesor realiza con cada } \\
\text { grupo intervenciones para } \\
\text { analizar y detectar el estado } \\
\text { de las tareas que están de- } \\
\text { sarrollando, orientando a los } \\
\text { estudiantes }\end{array}$ & 15 minutos & 85 minutos \\
\hline $\begin{array}{c}\text { PC } 1 \\
\text { (Puesta en } \\
\text { Común) }\end{array}$ & $\begin{array}{l}\text { Puesta en común de todas } \\
\text { las hipótesis de los trabajos } \\
\text { en grupo. } \\
\text { - Análisis, debate e influen- } \\
\text { cia en del trabajo de cada } \\
\text { grupo en el conjunto global } \\
\text { de la memoria de proyecto } \\
\text { propuesta }\end{array}$ & 20 minutos & 105 minutos \\
\hline $\begin{array}{c}\text { TC } 1 \\
\text { (Tareas en Casa) }\end{array}$ & $\begin{array}{l}\text { - } \text { Resumen de conclusiones. } \\
\text { realizar en horario fuera de } \\
\text { clase } \\
\text { - Programación de tareas a } \\
\text { realizar en la próxima sesión } \\
\text { de clase }\end{array}$ & 15 minutos & 120 minutos \\
\hline $\begin{array}{c}\text { R } 1 \\
\text { (Recompensa) }\end{array}$ & $\begin{array}{l}\text { Motivación de a los estu- } \\
\text { diantes en forma de recom- } \\
\text { pensa por la realización de } \\
\text { las tareas realizadas en clase } \\
\text { y fuera del aula. Recom- } \\
\text { pensa en la calificación de la } \\
\text { asignatura }\end{array}$ & $\begin{array}{l}\text { Sin tiempo } \\
\text { asignado } \\
\text { previamente }\end{array}$ & \\
\hline $\begin{array}{c}\text { I C E } 1 \\
\text { (Intervenciones } \\
\text { de Contraste } \\
\text { Especificas) }\end{array}$ & $\begin{array}{l}\text { El profesor realiza con los } \\
\text { responsables de la clase y } \\
\text { de cada equipo intervencio- } \\
\text { nes para matizar lo tratado } \\
\text { en clase y orientar en el de- } \\
\text { sarrollo de las tareas }\end{array}$ & $\begin{array}{l}\text { Sin tiempo } \\
\text { asignado } \\
\text { previamente }\end{array}$ & \\
\hline
\end{tabular}




\section{Secuencia de actividades}

La estructura del Ciclo de Mejora, con una duración total de 7 horas, es la siguiente:

- Sesión 1a. 2 horas

- Sesión 2a: 2 horas

- Sesión 3a. 1 hora fuera de clase

- Sesión 4a.: 2 horas

Las sesiones que se imparten en la asignatura tienen una asignación de 2 horas, se imparten los jueves de 17.30 h a 19.30 h. y se aplica a la asignatura de Proyectos de 40 curso de Grado en Ingeniería Aeroespacial; en particular al grupo 1 en el aula 003 de la Escuela Técnica Superior de Ingeniería.

Las horas destinadas al CIMA son 7 horas. La fecha de inicio es el 8 de noviembre y la de finalización el 16 de noviembre. Esto incluye la recuperación de una sesión que no se pudo impartir en su día, prevista para el 9 de noviembre, y una hora extra prevista para la semana entre el 8 y 15, con una fecha límite de entrega de documentación del 13 de noviembre.

La fecha de inicio del CIMA ha sido el 8 de noviembre y la fecha de su finalización el 16 de noviembre de 2018.

\section{Cuestionarios de exploración}

Con objeto de valorar el proceso del CIMA se desarrolla el siguiente cuestionario. El cuestionario está vinculado al apartado del mapa de contenidos y preguntas clave.

- Pregunta 1 (P1).

En la actualidad y en los próximos años está previsto la construcción de grandes hubs aeroportuarios como el NAICM (Nuevo Aeropuerto Internacional de la Ciudad de México), el Istanbul Grand, Turquía, el Pekín Daxing, 
China, el Al Maktoum Dubai World,Dubai, el Nuevo Aeropuerto Internacional de Adís Abeba, Etiopia o el Central Europe 1, Italia, en general el objetivo consiste en alcanzar la ansiada cifra de 100 millones de pasajeros al año o incluso superarla.

En el caso del Nuevo Aeropuerto de Estambul recientemente inaugurado, con capacidad para atender hasta 90 millones de pasajeros al año y un coste de unos 7.500 millones de euros, tiene el potencial de superar a Londres Heathrow como el aeropuerto más ocupado de Europa, con planes para aumentar aún más la capacidad a 200 millones de pasajeros al año, una vez que se espere que esté finalizada toda su infraestructura en 2025.

¿Te ves participando en la construcción de una infraestructura de tal entidad?

Explica ¿cuál crees que sería tu papel? y ¿qué podrías aportar?

- Pregunta 1a (P1a).

¿Cómo consideras tu preparación académica para afrontar situaciones como las planteadas en la pregunta anterior?

- Pregunta 2 (P2).

En tu caso (Asignatura Proyectos) estás interviniendo en la resolución de una problemática relacionada con el ámbito aeroportuario, ¿cómo entiendes que debe ser la gestión de los recursos humanos y materiales dentro de tu organización para llevar a cabo la tarea encomendada?

- Pregunta 2a (P2a).

Eres participe en una de las áreas encargadas de desarrollar una de las partes de la Memoria del Proyecto que os han asignado, ¿cuál puede ser tu aportación desde el punto de vista personal y desde el punto de vista técnico para el desarrollo de vuestra parte?

- Pregunta 2b (P2b).

Jornadas de Formación e Innovación Docente del Profesorado | № 2 (2019) Esta obra se distribuye con la licencia Creative Commons Reconocimiento-NoComercial-SinObraDerivada 
Para desarrollar la parte de la Memoria que os corresponde en vuestra área correspondiente, ¿cómo os habéis organizado?, ¿cuáles son las tareas que os habéis asignado para llevarlas a cabo? y ¿cómo hacéis el seguimiento de vuestros avances?

- Pregunta 2c (P2C). ¿Cuáles consideras que han sido las dificultades que se te han planteado al desarrollar la parte de la Memoria asignada?, ¿cuáles crees que puedes resolver tú o tu área o necesitas otras ayudas externas?

- Pregunta 2d (P2d).

Una vez identificadas las dificultades que se han presentado, ¿qué consideras que es necesario para resolverlas y que puede estar a tu alcance y en tu área?

- Pregunta 3 (P3). ¿Cómo suplirías tus carencias en las tareas que has de abordar para el desarrollo de tu parte de la Memoria?

- Pregunta 4 (P4).

Para llegar a una solución definitiva en la Memoria del Proyecto, ¿cómo entiendes que será tu interacción o la de tu área con el resto de áreas del Proyecto?

\section{Aplicación del ciclo de mejora}

\section{Desarrollo de las sesiones}

El contexto en el que se desarrollan las sesiones ya es favorable en cuanto a que la particularidad de la asignatura, parte práctica, permite desarrollar un campo con más posibilidades que una clase más teórica

Así mismo el hecho de disponer un grupo de alumnos reducido en torno a 20, permite una dedicación por parte del profesor sobre los alumnos que condiciona el seguimiento más personal de los alumnos. 
Se informó a los alumnos de su participación en el CIMA y aunque les resulto extraño, no parece que mostrarán mucho interés, si bien es cierto que algunos indicaron que no habían tenido ninguna asignatura en su Grado que se impartiera de esa manera.

\section{Evaluación del aprendizaje}

Indicar en primer lugar que el desarrollo del CIMA ha estado, en cuanto a los tiempos, muy ajustado. Han quedado pendientes algunos aspectos que hubiera deseado tratar con mayor profundidad pero que por motivos de agenda y horario sólo se han podido trabajar de forma inicial. Tomo nota de la situación para que sea mejorada en próximos ciclos de mejora.

Para la valoración de la evolución del proceso de enseñanza-aprendizaje de los alumnos planteo los niveles de aprendizaje que se indican en la siguiente tabla.

Tabla 4. Niveles de la escalera de aprendizaje

\begin{tabular}{|l|l|}
\hline \multicolumn{2}{|c|}{ Niveles de la Escalera de Aprendizaje } \\
\hline Niveles & \multicolumn{1}{c|}{ Corresponde a alumnos que: } \\
\hline Nivel 1 & $\begin{array}{l}\text { No poseen ningún conocimiento sobre la materia } \\
\text { Responden en blanco o sin sentido }\end{array}$ \\
\hline Nivel 2 & $\begin{array}{l}\text { Poseen un conocimiento inicial de carácter general e } \\
\text { intuitivo }\end{array}$ \\
\hline Nivel 3 & $\begin{array}{l}\text { Poseen un conocimiento concreto y utilizan algunas } \\
\text { habilidades para desarrollar el trabajo }\end{array}$ \\
\hline Nivel 4 & $\begin{array}{l}\text { Amplían el conocimiento necesario para el desarrollo del } \\
\text { trabajo y establecen relaciones entre contenidos, tomas } \\
\text { decisiones, realizan aportaciones propias y gestionan los } \\
\text { recursos de los que disponen }\end{array}$ \\
\hline
\end{tabular}

Los resultados del cuestionario inicial quedan reflejados en la siguiente tabla. 
Tabla 5. Resultado cuestionario inicial asignatura Proyectos

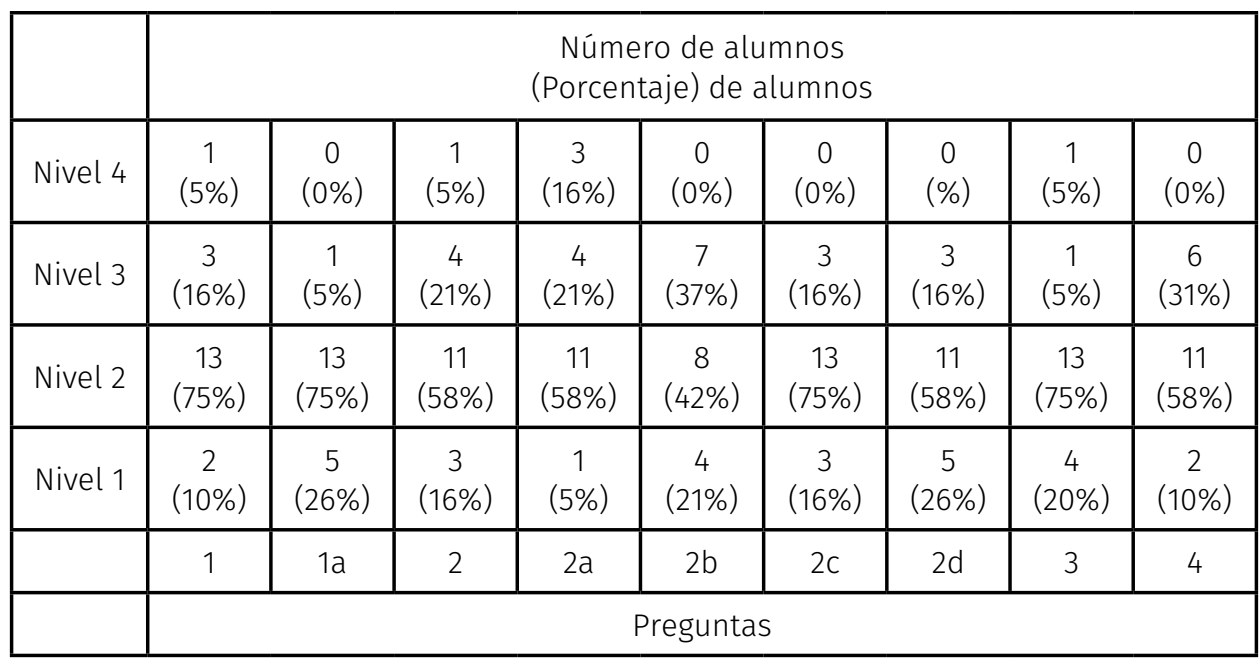

El resultado del cuestionario final queda resumido en la siguiente tabla.

Tabla 6. Resultado cuestionario final asignatura Proyectos

\begin{tabular}{|c|c|c|c|c|c|c|c|c|c|}
\hline & \multicolumn{10}{|c|}{$\begin{array}{c}\text { Número de alumnos } \\
\text { Porcentaje) de alumnos }\end{array}$} \\
\hline Nivel 4 & $\begin{array}{c}4 \\
(21 \%)\end{array}$ & $\begin{array}{c}2 \\
(10 \%)\end{array}$ & $\begin{array}{c}5 \\
(26 \%)\end{array}$ & $\begin{array}{c}11 \\
(58 \%)\end{array}$ & $\begin{array}{c}7 \\
(37 \%)\end{array}$ & $\begin{array}{c}3 \\
(16 \%)\end{array}$ & $\begin{array}{c}2 \\
(10 \%)\end{array}$ & $\begin{array}{c}6 \\
(31 \%)\end{array}$ & $\begin{array}{c}2 \\
(10 \%)\end{array}$ \\
\hline Nivel 3 & 4 & 1 & 8 & 5 & 8 & 3 & 1 & 7 & 3 \\
$(21 \%)$ & $(5 \%)$ & $(42 \%)$ & $(26 \%)$ & $(42 \%)$ & $(16 \%)$ & $(5 \%)$ & $(37 \%)$ & $(16 \%)$ \\
\hline Nivel 2 & $\begin{array}{c}11 \\
(58 \%)\end{array}$ & $\begin{array}{c}13 \\
(75 \%)\end{array}$ & $\begin{array}{c}5 \\
(26 \%)\end{array}$ & $\begin{array}{c}3 \\
(16 \%)\end{array}$ & $\begin{array}{c}4 \\
(21 \%)\end{array}$ & $\begin{array}{c}11 \\
(58 \%)\end{array}$ & $\begin{array}{c}11 \\
(58 \%)\end{array}$ & $\begin{array}{c}5 \\
(26 \%)\end{array}$ & $\begin{array}{c}11 \\
(58 \%)\end{array}$ \\
\hline Nivel 1 & 0 & 2 & 1 & 0 & 0 & 2 & 5 & 1 & 2 \\
$(0 \%)$ & $(10 \%)$ & $(5 \%)$ & $(0 \%)$ & $(0 \%)$ & $(10 \%)$ & $(26 \%)$ & $(5 \%)$ & $(10 \%)$ \\
\hline & 1 & $1 \mathrm{a}$ & 2 & $2 \mathrm{a}$ & $2 \mathrm{~b}$ & $2 \mathrm{c}$ & $2 \mathrm{~d}$ & 3 & 4 \\
\hline & \multicolumn{10}{|c|}{ 26reguntas } & & & \\
\hline
\end{tabular}

El grado de participación de los alumnos ha sido del 100\%, (han asistido de forma regular 19 alumnos por sesión) aunque algunos, dos de ellos, no han cumplimentado satisfactoriamente las preguntas. 
En lo referente a la evaluación del aprendizaje de los alumnos y teniendo en cuenta que antes de la aplicación del CIMA, el desarrollo de la clase es conforme a lo que se pretende en el CIMA, los alumnos parten con una cierta "ventaja" de lo que se espera de ellos; y es por ello por lo que en general en casi todas las preguntas el nivel 1 se ha superado con bastante facilidad.

Tras la aplicación del CIMA, en todas las preguntas se pueden observar cambios de niveles de unas preguntas a otras. Hay contenidos especialmente los trasversales que no han sido capaces de identificar y de aplicar y que afectan a todo el CIMA.

En general la evaluación del CIMA ha sido positiva y aunque no se ha alcanzado un alto número/porcentaje de estudiantes en el nivel 4 en todas las preguntas planteadas, la apreciación general es que se han alcanzado valores significativos en los niveles 2 y 3; lo que permite estar satisfecho con la aplicación del CIMA.

Como aspecto a mejorar en la aplicación de CIMA, destacar que el ritmo de desarrollo del CIMA no ha sido todo lo ágil que se pretendía, llegando en algunos aspectos a generarse situaciones de "bloqueo" que no permitían avanzar en el desarrollo del trabajo de forma adecuada, y que repercutía sobre todo en algunos alumnos que por ello se sentían algo agobiados y frustrados porque veían que no se avanzaba; pero lo que no percibían es que esa situación es la que debían de tratar de solventar y enfrentarse a ella para dar solución al problema planteado.

Por último, dejar constancia de que el resultado obtenido ha sido satisfactorio porque ha permitido que los alumnos identifiquen los aspectos que les han sido requeridos, pero también indicar que se puede mejorar en las siguientes líneas de trabajo: 
- Establecer una dinámica, apoyada en tareas que permitan a los estudiantes, centrarse en los aspectos que se pretenden potenciar.

- Realizar un seguimiento más riguroso de las hipótesis adoptadas por los alumnos, con el fin de orientar y evitar situaciones de "bloqueo".

- Establecer pautas para motivar y atraer la atención de los alumnos por el ámbito de la asignatura.

\section{Evaluación del CIMA}

Como ya he indicado, en líneas generales se ha mejorado la dinámica del CIMA respecto al impartido en el curso anterior 2017-2018, pero se ha constatado que es necesario ampliar el tiempo asociado a los objetivos planteados.

Es posible hacer mejoras en la implicación y en la participación de los alumnos, considerando e incorporando en el proceso de enseñanza-aprendizaje la frustración que pueden sentir los estudiantes al percibir que no se avanza en el objetivo u objetivos propuestos, y trasladándoles la importancia de terminar el trabajo lo antes posible ya que el tiempo de ejecución del proyecto es determinante.

El desarrollo del CIMA me ha permitido tener en cuenta otro aspecto a valorar: evitar frustraciones por no alcanzar objetivos y hacerles ver a los estudiantes que quizás no se puedan alcanzar en su totalidad pero que se está en el camino y que se están poniendo los cimientos para alcanzarlos, aunque no sea por completo.

Otro aspecto de la experiencia que se puede incorporar en la práctica docente es la consideración de que a pesar de delegar parte del desarrollo de las tareas en los alumnos y solicitarles su implicación y trabajo, resulta de gran importancia que los profesores guíen a los alumnos de forma constante. Hay que estar "pinchándoles" para que no bajen la guardia. Lo denominaría exigirles desde el cariño. 


\section{Referencias bibliográficas}

Bain, K. (2004). What the Best Collage Teachers Do? Cambridge, MA: Harvard University Press. (Trad. cast.: Lo que hacen los mejores profesores universitarios. Valencia: Publicaciones de la Universidad de Valencia, 2005).

Finkel, D. (2000). Teaching with Your Mouth Shut. Portsmouth, NH: Heinemann Boynton/Cook. (Trad. cast.: Dar clase con la boca cerrada. Valencia: Publicaciones de la Universidad de Valencia, 2008)

Fernández de la Cruz, J. J. (2017). Desarrollo Memoria Proyecto Constructivo. CMD, III PPD. Instituto de Ciencias de la Educación de la Universidad de Sevilla.

Herrera-Martín, J. A. (2016). ¿Cómo implantamos las instalaciones en el edificio? Representación gráfica, trazado y dimensiones. En R. Porlán y E. Navarro (Coords.), III Jornadas de Docencia Universitaria (págs. 730-745). Sevilla: Instituto de Ciencias de la Educacion de la Universidad de Sevilla.

Herrera-Martín, J. A. (2017). ¿Cuánto vale el suelo, una vivienda o un local? En R. Porlán y E. Navarro (Coords.), IV Jornadas de Docencia Universitaria (págs. 937-956). Sevilla: Instituto de Ciencias de la Educación de la Universidad de Sevilla.

Jornadas de Formación e Innovación Docente del Profesorado | № 2 (2019) Esta obra se distribuye con la licencia Creative Commons Reconocimiento-NoComercial-SinObraDerivada Internacional (CC BY-NC-ND 4.0.) 